\title{
Harnessing the mode mixing in optical fiber-tip cavities
}

\author{
Nina Podoliak ${ }^{1}$, Hiroki Takahashi ${ }^{2}$, Matthias Keller ${ }^{2}$, Peter \\ Horak $^{1}$ \\ ${ }^{1}$ Optoelectronics Research Centre, University of Southampton, Southampton SO17 \\ 1BJ, U.K. \\ ${ }^{2}$ Department of Physics and Astronomy, University of Sussex, Falmer, BN1 9QH, \\ U.K. \\ E-mail: N.Podoliak@soton.ac.uk
}

\begin{abstract}
We present a systematic numerical study of Fabry-Pérot optical cavities with Gaussian-shape mirrors formed between tips of optical fibers. Such cavities can be fabricated by laser machining of fiber tips and are promising systems for achieving strong coupling between atomic particles and an optical field as required for quantum information applications. Using a mode mixing matrix method, we analyze the cavity optical eigenmodes and corresponding losses depending on a range of cavity-shape parameters, such as mirror radius of curvature, indentation depth and cavity length. The Gaussian shape of the mirrors causes mixing of optical modes in the cavity. We investigate the effect of the mode mixing on the coherent atom-cavity coupling as well as the mode matching between the cavity and a single-mode optical fiber. While the mode mixing is associated with increased cavity losses, it can also lead to an enhancement of the local optical field. We demonstrate that around the resonance between the fundamental and $2^{\text {nd }}$ order Laguerre-Gaussian modes of the cavity it is possible to obtain $50 \%$ enhancement of the atom-cavity coupling at the cavity center while still maintaining low cavity losses and high cavity-fiber optical coupling.
\end{abstract}




\section{Motivation}

In the ongoing search for novel systems to strongly couple atomic particles to an optical field, especially in the context of quantum information networks $[1,2,3,4]$ and quantum sensors [5, 6], fiber-tip cavities have attracted significant attention. Laser machined end facets of optical fibers with high reflective coatings have been proposed to use as cavity mirrors and employed in various experiments with atoms and ions [7, 8, 9, 10, 11, 12]. The potential applications of fiber tip cavities have led to several recent publications on novel laser machining methods $[13,14]$ and on mode matching between the cavity mode and the output mode of the optical fiber $[15,16]$. In contrast to conventional spherical mirrors, fiber-tip cavities usually have Gaussian-shaped surfaces, imprinted by the intensity distribution of the machining laser. Even though for small separations between the cavity mirrors (much smaller than the radius of curvature) the mirror shapes can be considered as approximately spherical, for longer cavity lengths this is no longer appropriate. For long cavities, and thus larger cavity mode field diameters at the mirrors, the deviation from the spherical shape leads to mode mixing with associated increase of optical losses [17]. Whereas this is usually an unwanted effect, in this article we show that mode mixing can be utilized to enhance the coupling strength between the cavity mode and atomic particles trapped at the cavity center. Eigenmodes of cavities with Gaussian-shaped mirrors can exhibit strong enhancement of the cavity field in the cavity center, leading to significantly increased coupling compared to spherical cavities. In particular, we investigate the effect of mode mixing in cavities with Gaussian-shaped mirrors on the cavity losses, the coherent coupling strength and the mode matching efficiency between the cavity and the output mode of a single-mode optical fiber.

The coherent coupling between a particle, such as a cold ion or atom, located at a coordinate $\mathbf{r}$ in an cavity with an optical field mode $\Psi(\mathbf{r})$ is characterized by the coupling strength

$$
g(\mathbf{r})=\sqrt{\frac{\pi \mu^{2} c}{\hbar \epsilon_{0} \lambda L}} \Psi(\mathbf{r}) \equiv g_{0} \Psi(\mathbf{r}),
$$

where $\mu$ is the transition dipole moment of the particle, $\lambda$ its transition wavelength, $L$ is the cavity length, and the cavity mode field $\Psi(\mathbf{r})$ is normalized to satisfy $\int_{S} d^{2} r|\Psi(\mathbf{r})|^{2}=1$ at any cross-section. If the particle is located at the center of the cavity the coupling strength reduces to

$$
g(0)=g_{0} \Psi(0) \text {. }
$$

To achieve strong atom-cavity coupling, the coherent coupling rate $g(0)$ between the atomic particle and the cavity must be larger than the strength of any incoherent processes, such as the spontaneous decay rate $\Gamma$ of the atomic particle and the loss rate $\kappa$ of the cavity field, i. e., the cooperativity parameter defined as

$$
C=\frac{g(0)^{2}}{\kappa \Gamma}
$$


must be larger than one. One of the methods to increase the coupling strength is to decrease the cavity length. However, coupling atomic particles to an optical fiber tip cavity often imposes constrains on the cavity length due to the requirement for optical side access, delivery of atoms into the fiber cavity and integration of trapping structures into the cavity. These limitations increase the minimal cavity length and thus reduce the maximum coupling strength between the atomic particle and the fiber cavity whilst maintaining low cavity losses. This is in particular true for combining optical cavities with single ions held in a radio-frequency trap, as the dielectric mirrors forming the cavity in the close vicinity of the ion trap electrodes can distort the ion trapping fields $[18,19]$. Another method is to increase the field amplitude in the center of the cavity $\Psi(0)$. Here, we numerically explore the latter method by taking advantage of the Gaussian profile of the cavity mirrors which gives rise to highly localized cavity modes.

This paper is organized as follows. First, in Sec. 2, we describe our theoretical and numerical model. In Sec. 3 we present the results of our simulations, in particular on mode field amplitudes, optical losses and mode mixing in the cavity in Sec. 3.2 and on output coupling efficiency into optical fiber in Sec. 3.3. Finally we summarize and conclude in Sec. 4.

\section{Modeling}

\subsection{Cavity geometry}

We consider a symmetric optical cavity formed by two identical concave mirrors with a Gaussian profile as fabricated by laser ablation $[7,13]$ on the tips of optical fibers, as sketched in figure 1. The concave profile of the mirror is characterized by two parameters: the radius of curvature at the center, $R$, and the indentation depth, $D$, with the radial

profile depth of the mirror being $d(r)=D \exp \left\{-\frac{r^{2}}{2 R D}\right\}$, where $r$ is the radial distance from the mirror center. The cavity length is $L$, the wavelength of the optical mode is $\lambda$.

For the simulations in Sec. 3 we choose the following system parameters. The mirror profile has a radius of curvature $R=300,500$ or $700 \mu \mathrm{m}$. The depth $D$ varies from 2 to $10 \mu \mathrm{m}$. The fiber external radius is $R_{a}=100 \mu \mathrm{m}$. These parameters correspond to the mirrors experimentally fabricated by the modified laser oblation method as presented in Ref. [13]. The cavity length $L$ varies from a few $\mu \mathrm{m}$ up to $2 R$. The modes are calculated at $\lambda=866 \mathrm{~nm}$, the resonant wavelength of $\mathrm{Ca}^{+}$ions frequently used in ion trap experiments.

\subsection{Modeling method}

Here we follow the approach of Ref. [20] to calculate the eigenmodes of the fiber cavity. Optical mode fields, $\Psi_{i}(\mathbf{r})$, of a cavity with a Gaussian profile mirror can be expressed as a superposition of a given set of basis modes, $\left\{\psi_{n}(\mathbf{r})\right\}$,

$$
\Psi_{i}(\mathbf{r})=\sum_{n} C_{i, n} \psi_{n}(\mathbf{r})
$$




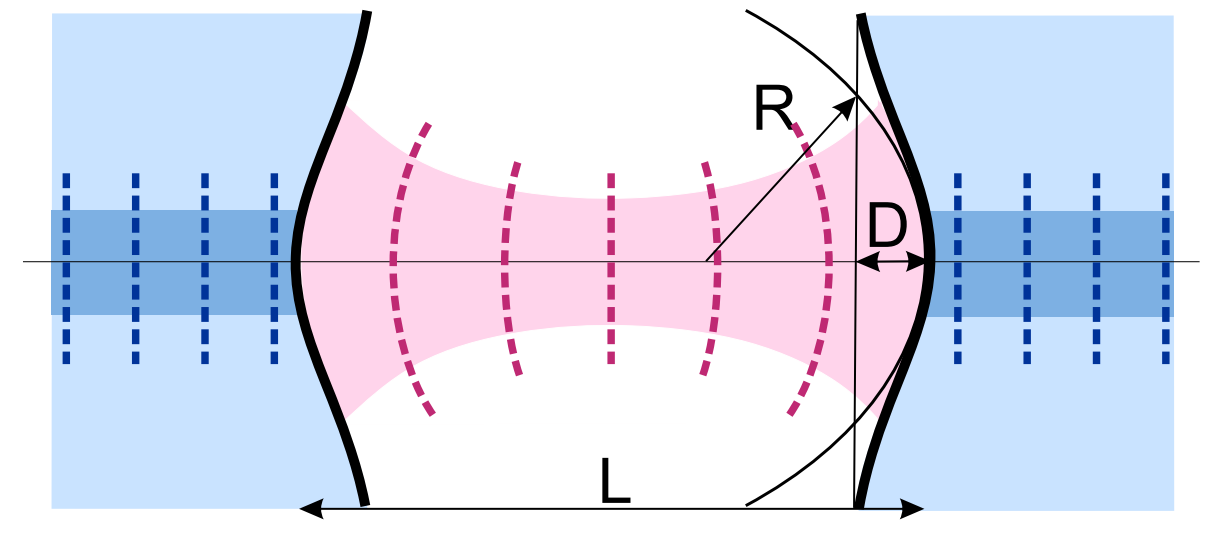

Figure 1. Schematic of a fiber tip cavity with Gaussian-shaped mirrors. Also indicated are the cavity and fiber modes (red and blue shading) and their wave fronts (dashed lines). $L$ is the cavity length, $R$ is the radius of mirror curvature at the center, and $D$ is the indentation depth.

We will consider only linearly polarized modes, thus the optical field can be described by a scalar field $\Psi_{i}(\mathbf{r})$. Due to the rotational symmetry of the cavity and its close resemblance to a cavity with spherical mirrors we use the Laguerre-Gaussian modes as the basis modes. Moreover, we asume cylindrical symmetry of the system and thus restrict our model to the radially symmetric basis modes

$$
\psi_{n}^{ \pm}(\rho, \zeta)=\sqrt{\frac{2}{\pi}} L_{n}\left(2 \rho^{2}\right) \exp \left\{-\rho^{2} \pm i\left(-\zeta \rho^{2}+(2 n+1) \tan ^{-1} \zeta\right)\right\}
$$

where $\rho=r / w(\zeta)$ and $\zeta=z / z_{0}$ are the dimensionless radial and axial coordinates, $w(\zeta)=w_{0} \sqrt{1+\zeta^{2}}$ is the basis mode radius, $w_{0}$ is the mode waist (minimal mode radius), $z_{0}=k w_{0}^{2} / 2$ is the Rayleigh length, $k=2 \pi / \lambda$ is the wave number, $L_{n}$ are Laguerre polynomials and $n$ is the mode order. The index \pm marks the propagation direction. The longitudinal phase $\exp \{ \pm i k z\}$ is not included in the basis mode field but will be included explicitly.

The change of an optical mode undergoing one round trip through the cavity can be represented by a mode-mixing operator $M$. Then finding the cavity eigenmodes reduces to solving the eigenproblem $[17,20]$

$$
\gamma_{i} \Psi_{i}=M \Psi_{i}
$$

where the eigenvectors are the cavity modes and the eigenvalues $\gamma_{i}$ define the field amplitude changes of the corresponding modes acquired per round trip. Thus, the loss per round trip $\mathcal{L}_{i}$ for the mode $\Psi_{i}$ can be evaluated as

$$
\mathcal{L}_{i}=1-\left|\gamma_{i}\right|^{2} .
$$

The mode-mixing matrix of the cavity is given by

$$
M=\exp \{2 i k L\} B^{+} B^{-},
$$

where the exponent represents the longitudinal phase shift acquired over the cavity round trip. The matrices $B^{ \pm}$represent the right- and left-side mirrors of the cavity 
positioned at coordinates $z= \pm L / 2$, respectively, and are given by the mode overlap integrals taken over the finite extent of the mirrors,

$$
B_{n, m}^{ \pm}=\int_{0}^{\rho_{a}} \psi_{n}^{\mp} \psi_{m}^{ \pm *} \exp \{-2 i k|\Delta(\rho)|\} 2 \pi \rho d \rho
$$

where $\rho_{a}=R_{a} / w\left(\zeta_{m}\right)$ with the mirror radius $R_{a}$ and the axial mirror position $\zeta_{m}= \pm \frac{L}{2 z_{0}}$. $\Delta(r)$ describes the deviation of the mirror profile from a plane surface, which in our case is

$$
\Delta(r)=D\left(1-\exp \left\{-\frac{r^{2}}{2 R D}\right\}\right)
$$

The computation of the cavity modes consists of the following steps. First, a suitable basis $\left\{\psi_{n}\right\}$ is found for each cavity length, which is defined by the waist $w_{0}$ of the fundamental basis mode. As we are mainly interested in the lowest order cavity mode, the waist $w_{0}$ is chosen by maximizing the $M_{00}$ element matrix $M$ calculated using equations (8) and (9) with the size of the basis set of $\mathrm{N}$ modes. The full matrix $M$ is then calculated for the basis set (5) with this value of $w_{0}$. We found that simulations with $N>20$ deviate by less than one percent and thus use $N=30$ for all our simulations. The cavity modes are then found by solving the eigenproblem (6), where the eigenvectors contain the mode composition coefficients $C_{i, n}$ and the eigenvalues define the mode losses as per equation (7). Using the $C_{i, n}$ coefficients, the field profile of any mode at any point in the cavity can be found using equation (4).

\section{Results and discussion}

\subsection{Cavity optical modes and losses}

First we consider the optimal waist of the basis modes $w_{0}$ as described above for cavities with different mirror profile parameters. As we will show later, $w_{0}$ corresponds to the waist of the fundamental mode apart from cavities with lengths close to some discrete resonances with increased mode mixing. The optimal waist for cavities with different radii of curvature and the same depth, and for the same radius of curvature and different depths are compared in figure 2(a) and (b), respectively. The results are also compared to the mode waist of a cavity consisting of ideal spherical mirrors with radius of curvature $R=500 \mu \mathrm{m}$. Here we can identify the scaling factor for the waist size as $w / R^{1 / 2}$, and for the cavity length as $L / R$. With this normalization, the deviation of the scaled waist size from the mode waist of a spherical cavity depends only on the mirror depth parameter $D$ but not on $R$. This dependence can be explained by considering the expansion of the Gaussian profile of the mirror given by equation (10) in paraxial approximation

$$
\Delta(r)=D\left(1-\exp \left\{-\frac{r^{2}}{2 R D}\right\}\right) \approx \frac{r^{2}}{2 R}-\frac{r^{4}}{8 R^{2} D},
$$

where the first term describes the spherical profile with the radius of curvature $R$, and the second term defines the deviation of the Gaussian shape from the spherical profile. As the radial coordinate $r$ scales with $R^{1 / 2}$, the deviation of the Gaussian mirror from the 
spherical one in the first approximation is inversely proportional to the depth parameter $D$.

For further comparison with the spherical cavity we also introduce an effective radius of curvature for the equivalent spherical mirror with the same mode waist $w_{0}$ as $R_{\text {eff }}=\frac{L}{2}+\frac{2 \pi^{2} w_{0}^{4}}{\lambda^{2} L}$ (see figure $3(\mathrm{c})$ and $(\mathrm{d})$ ). The deviations of the mode waist size and the effective radius of curvature from the ideal spherical mirror case are larger for smaller $D$, which is consistent with the fact that for Gaussian-shaped mirrors with smaller $D$ the profile deviates more strongly from a spherical shape at smaller radius $r$, see equation (11). The deviations from the spherical mirror cavity become pronounced for $L / R \geq 1$, i. e., for confocal to concentric cavity geometries. Moreover, we note that the effective radius of curvature increases with increasing cavity length which, in principle, allows formation of a stable cavity mode for cavity lengths above $2 R$.

Similarly, for $L<R$ the cavity mode $\Psi_{0}$ almost entirely consists of the fundamental $\psi_{0}$ mode. However, at $L \gtrsim R$, mode mixing with higher order modes occurs, in particular around certain discrete cavity lengths. The mode composition coefficients of the lowest order mode $\Psi_{0}$ of the cavity with $R=500 \mu \mathrm{m}$ and $D=5 \mu \mathrm{m}$ are shown in figure 3(a). The most prominent of these resonances are marked by light blue dashed lines in the figure. The first three lines mark resonances that involve mainly the fundamental and
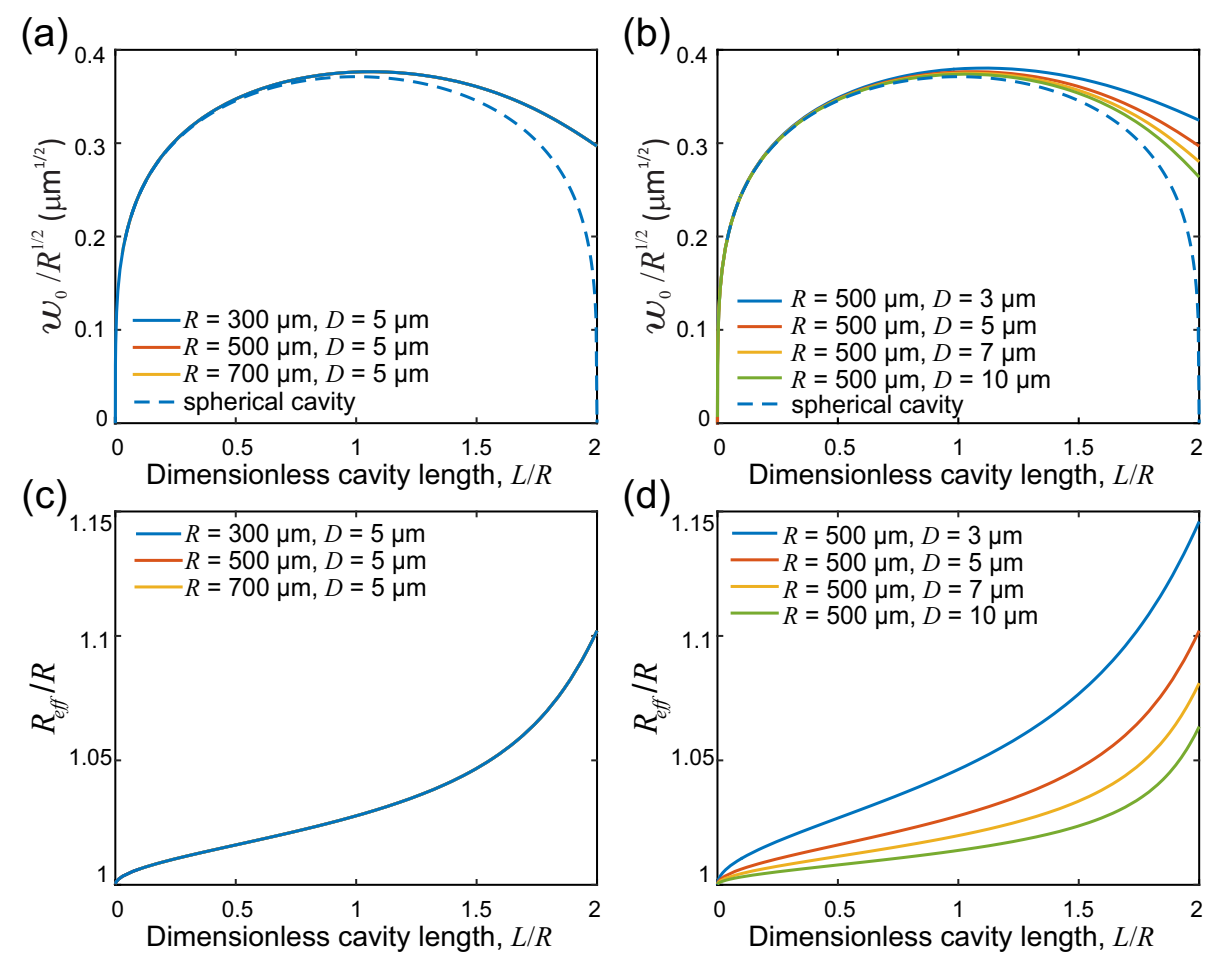

Figure 2. (a) Optimized waist of basis modes $w_{0}$ for cavities with different radii of curvature $R$ and the same depth $D=5 \mu \mathrm{m}$ (note that all three curves coincide). (b) $w_{0}$ for cavities with $R=500 \mu \mathrm{m}$ and different depths $D$. (c) Effective radius of curvature $R_{\text {eff }}$ corresponding to (a). (d) $R_{\text {eff }}$ corresponding to (b). The dashed lines in (a) and (b) are the mode waist of a cavity with spherical mirrors. 
the $2^{\text {nd }}, 4^{\text {th }}$ and $6^{\text {th }}$ order modes with a small contribution of the higher order modes, respectively. Due to the $\rho$ dependence of the mode-mixing matrix (8), only modes with the same parity mix. Apart from the fundamental order mode that mainly mixes with the lowest even order modes, the $1^{\text {st }}$ order mode has the strongest resonances with the $3^{\text {th }}$ and $5^{\text {th }}$ order modes, etc.

The loss for the fundamental cavity mode calculated for cavities with different radii of curvature and the same depth $D=5 \mu \mathrm{m}$ as a function of the dimensionless cavity length is plotted in figure 3(b). Whenever mode mixing occurs, the losses increase drastically, resulting in sharp peaks in the round trip loss. This is due to the increased mode diameters of the higher order mode that lead to larger clipping losses on the mirrors. Here we should stress that the mode composition coefficients as well as the loss of the cavities that have the same depth $D$ coincide in the dimensionless coordinates and the small differences seen in figure 3(b) are due to the numerical resolution of our simulations. Mixing between the fundamental and higher order modes also occurs at the same dimensionless cavity lengths. However, this is not the case for cavities with different depth parameters. Figure 4 shows the $\left|C_{00}\right|$ coefficient of the lowest order mode and corresponding round trip loss of cavities with the same radius of curvature $R=500 \mu \mathrm{m}$ and different depths. The losses increase with increasing cavity length

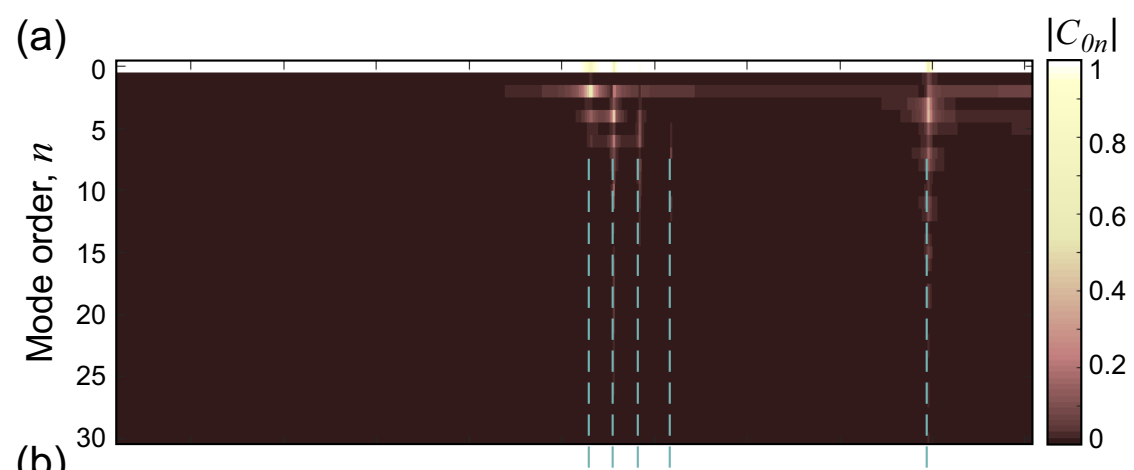

(b)

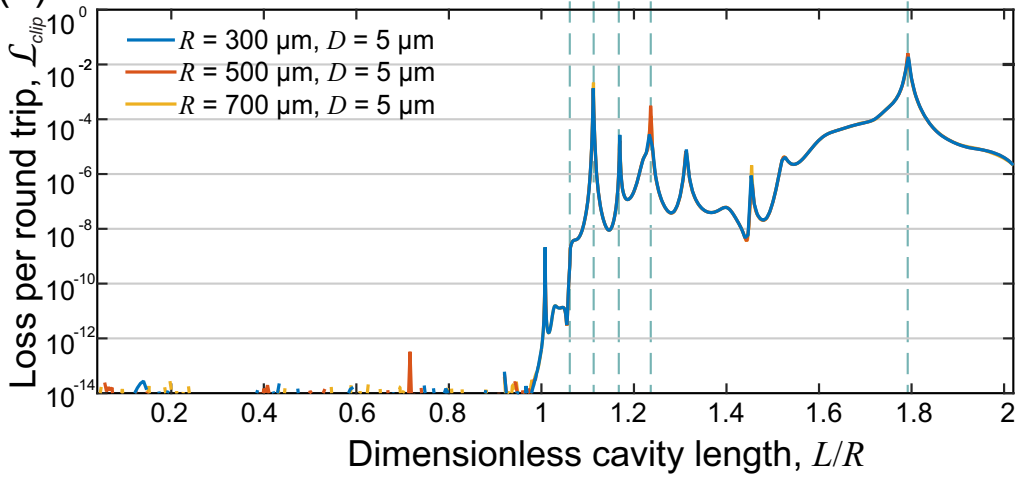

Figure 3. (a) Absolute value of the composition coefficients $C_{0 n}$ of the fundamental mode of a cavity with $R=500 \mu \mathrm{m}$ and $D=5 \mu \mathrm{m}$ versus cavity length $L$. (b) Loss per round trip of the fundamental mode of cavities with different $R$ and the same depth $D=5 \mu \mathrm{m}$ versus $L$ (note that all three curves coincide; the differences between curves are due to numerical resolution). Dashed lines mark regions where strong mode mixing occurs. 
(a)

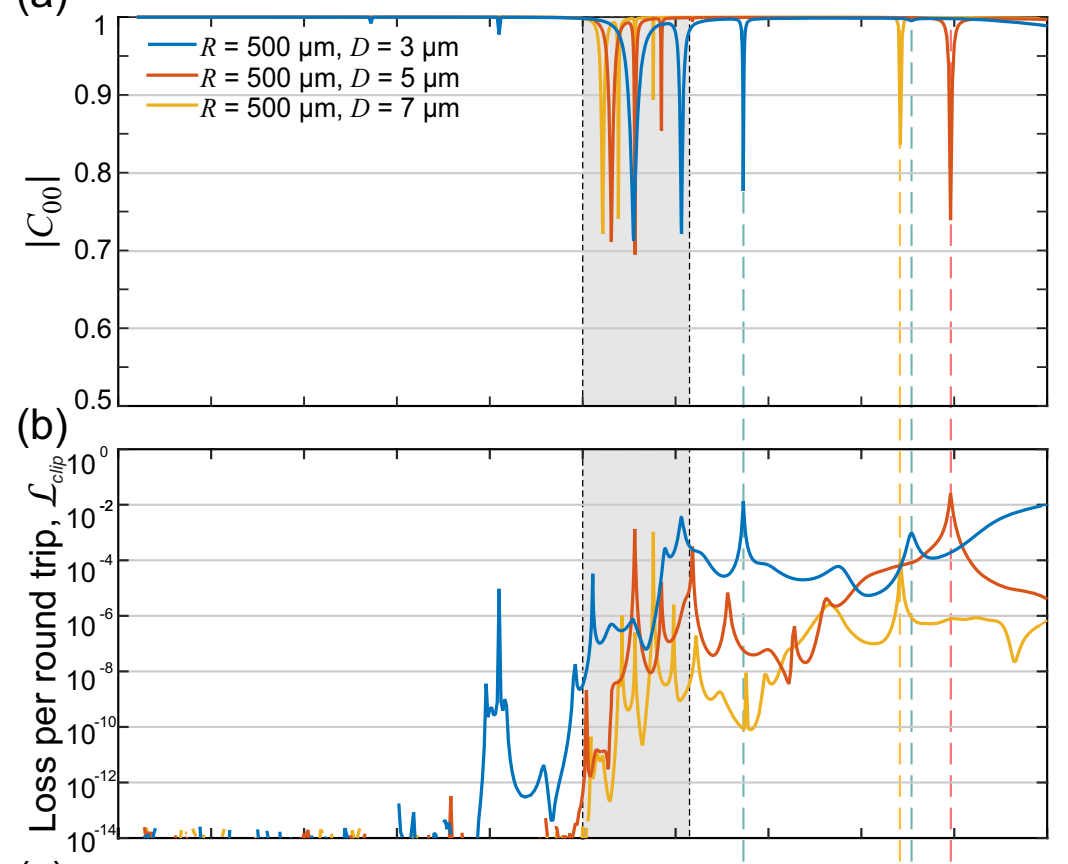

(b)

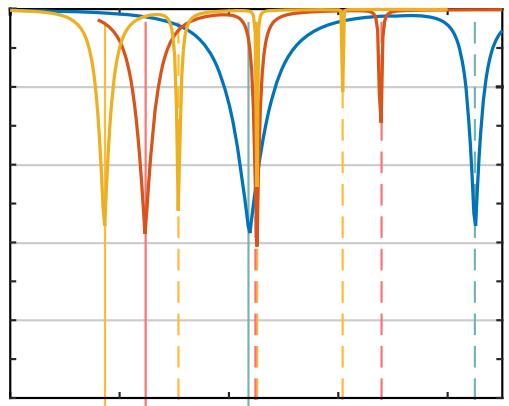

(c)

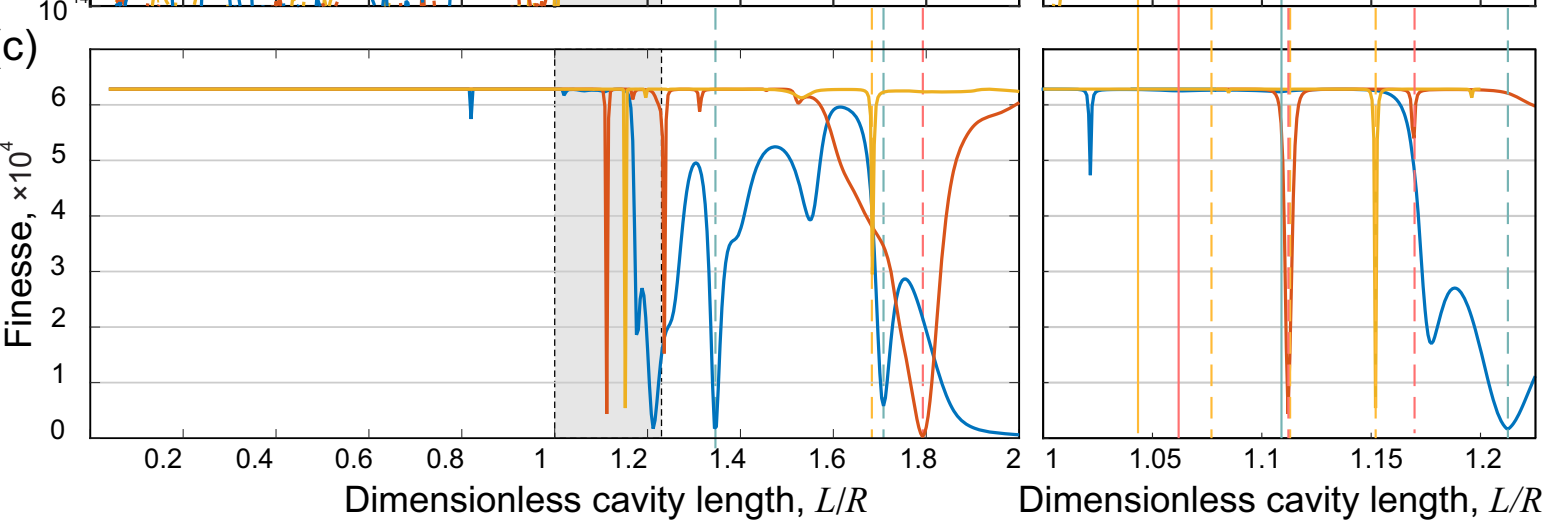

Figure 4. (a) $\left|C_{00}\right|$ coefficient of the fundamental mode of a cavity with the same radius of curvature $R=500 \mu \mathrm{m}$ and different depths $D$ as a function of dimensionless cavity length. (b) Corresponding loss per round trip. (c) Cavity finesse comprising the mode loss of (b) and mirror losses of $10^{-4}$ per round trip. The shaded regions between the dotted black lines are magnified on the right-hand side plots. Vertical solid and dashed lines mark regions where mixing with the $2^{\text {nd }}$ or higher order modes occurs.

and are in general higher for cavities with smaller depths $D$. Mode mixing between the fundamental and the $2^{\text {nd }}, 4^{\text {th }}$ and $6^{\text {th }}$ order modes also occurs at shorter cavity lengths and the distances between resonance peaks are smaller in cavities with larger $D$ parameters.

The quality of an optical cavity is often characterized by its finesse $\mathcal{F}$ which depends on the total loss consisting of the clipping loss of the optical mode upon reflection from the mirrors $\mathcal{L}_{\text {clip }}$ and the transmission and absorption loss of the mirrors $\mathcal{L}_{\text {mir }}$ :

$$
\mathcal{F}=\frac{2 \pi}{\mathcal{L}_{\text {clip }}+\mathcal{L}_{\text {mir }}}
$$

For typical mirrors fabricated on fiber tips, the loss is of the order of $10^{-4}$ [17], which 
should allow for a cavity finesse above $6 \times 10^{4}$. As can be seen in figure 3 and 4 , the resonances that involve higher order modes are usually associated with an increase of losses, which may be a hindrance for a high finesse of this cavity mode [17]. However, when a resonance occurs mainly with the $2^{\text {nd }}$ order mode, the loss per round trip is still much lower than the mirror loss (below $10^{-6}$ at $D=3 \mu \mathrm{m}$, and even lower for larger $D$ ), causing no decrease in the cavity finesse. In the next section we will study the cavity modes around this resonance in more detail.

\subsection{Mode mixing in the cavity}

We start by determining the cavity length at which the mode mixing between the fundamental and the $2^{\text {nd }}$ order mode occurs. At this resonance, the eigenvalues $\gamma_{i}$ of equation (6) have the same phase for both modes, i. e., the modes become degenerate. Figure 5(a) shows the phases of the $0^{\text {th }}$ and $2^{\text {nd }}$ order mode eigenvalues of the cavities characterized by different depth parameters. The corresponding phases of basis modes $\psi_{0}$ and $\psi_{2}$ are also shown in the figure. The factor of $\exp \{2 i k L\}$ is omitted in the phase to remove fast oscillations. As can be seen, mode mixing occurs in the region where the phases of the basis modes cross. A close examination shows a typical avoided crossing behavior between eigenmodes around the resonances. In cavities with larger $D$ parameters, the phases of basis modes cross at shorter cavity lengths, and the separation between the phases of the eigenmodes is smaller. This explains that the resonances in these cavities are sharper (see figure 4). Further deviation of the eigenmodes phases from the $0^{\text {th }}$ order mode line in figure $5(\mathrm{a})$ is related to the mixing with the $4^{\text {th }}$ order mode, not shown here. The length of the cavity at which the resonance between the $0^{\text {th }}$ and $2^{\text {nd }}$ order modes occurs, $L_{r}$, depending on the cavity depth $D$ is plotted in figure 5(b).

Next, we investigate the mode profile around the resonance. Here we will concentrate on a cavity with $R=500 \mu \mathrm{m}, D=5 \mu \mathrm{m}$, for which the resonance occurs at a cavity length $L \approx 531 \mu \mathrm{m}$. We should point out that the effects described below are not limited to these particular dimensions and are also observed at different cavity parameters $R$ and $D$ with corresponding scaling of the optical mode sizes and the resonant cavity length as discussed above. The cross-sections of the lowest order mode at the center and on the mirrors of the cavity away from the resonance $(L=500 \mu \mathrm{m})$ and at the resonance $(L=531 \mu \mathrm{m})$ are compared in figure 6 . Away from the resonance, the mode is approximately Gaussian as it is nearly entirely composed of the fundamental

basis mode $\psi_{0}$, i. e., $\left|C_{00}\right| \approx 1$ as seen in figures 3 and 4 . In this case the mode waist at the cavity center is given by the optimized basis mode waist $w_{0}$, as already indicated above. At the resonance, mode mixing occurs and the superposition of the coupled basis modes with the corresponding phases results in more complex mode profiles. Most importantly, the in-phase combination of the coupled basis modes creates an eigenmode with a small bright peak in the center of the cavity surrounded by a pale concentric ring, see figure $6(\mathrm{c})$. 
(a)

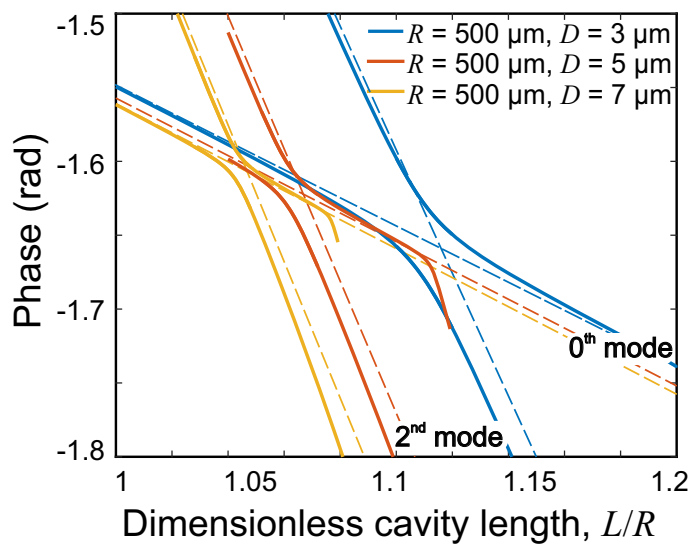

(b)

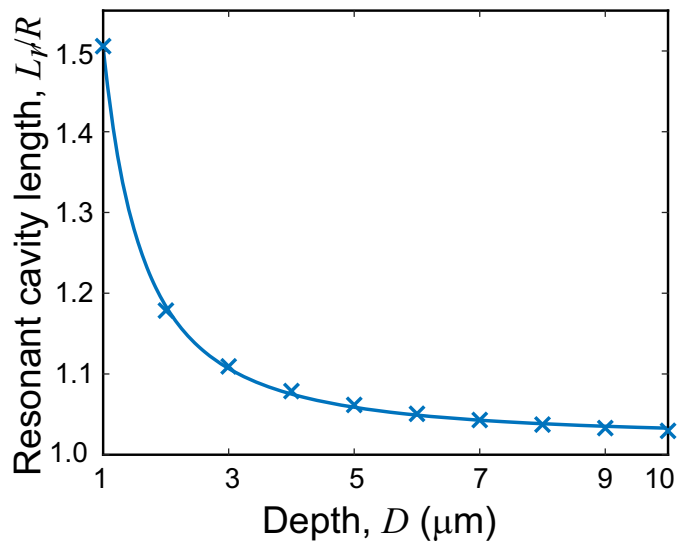

Figure 5. (a) Phase of the eigenvalue $\gamma_{i}$ of the fundamental ( $0^{\text {th }}$ order) and the $2^{\text {nd }}$ order eigenmodes (solid lines) and $0^{\text {th }}$ and $2^{\text {nd }}$ order basis modes (dashed lines) of cavities characterized by $R=500 \mu \mathrm{m}$ and different $D$ versus cavity length. (b) Resonant cavity length $L_{r}$ at which the mode mixing between the $0^{t h}$ and $2^{\text {nd }}$ order mode occurs versus $D$. Crosses are simulated data, the solid line is a numerical fit as a guide to the eye.
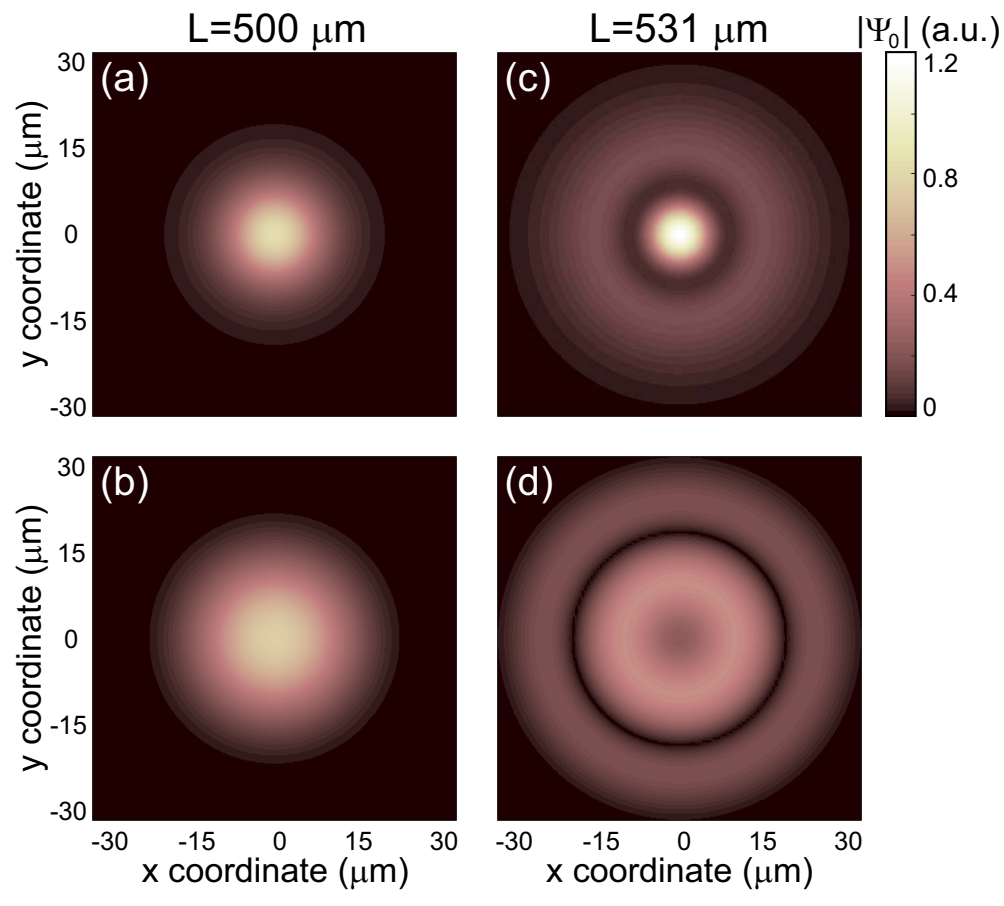

Figure 6. Lowest order mode field amplitude (a) and (c) at the center and (b) and (d) on the mirror planes for cavities with $L=500 \mu \mathrm{m}$ (away from the resonance) and with $L=531 \mu \mathrm{m}$ (at the resonance), respectively. Cavity parameters are $R=500 \mu \mathrm{m}$, $D=5 \mu \mathrm{m}$.

The mode field amplitude at the center of the cavity as a function of the cavity length in the region around the resonance is shown in figure 7 . As the cavity length approaches the resonant value, the field amplitude of the lowest order mode increases 


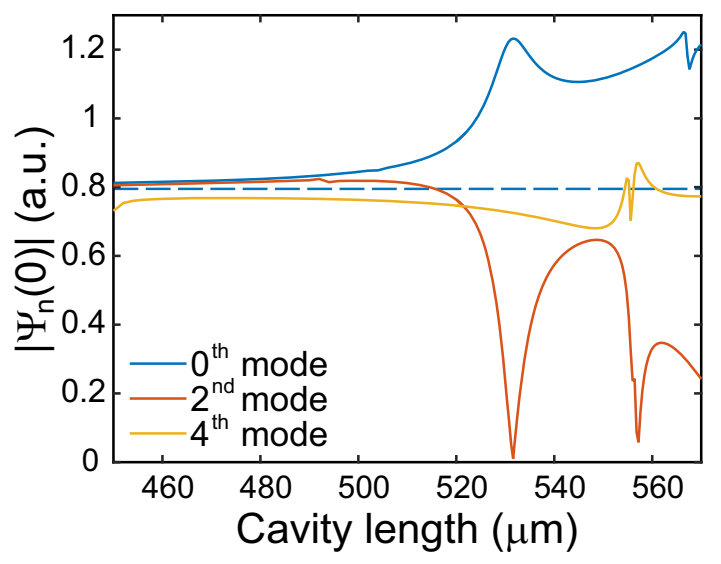

Figure 7. Amplitudes of the $0^{\text {th }}, 2^{\text {nd }}$ and $4^{\text {th }}$ modes at the center of the cavity with $R=500 \mu \mathrm{m}, D=5 \mu \mathrm{m}$ versus $L$ around the resonance at $L=531 \mu \mathrm{m}$. The dashed line shows the amplitude of the fundamental mode for a spherical cavity with $R=500 \mu \mathrm{m}$, which changes by less than $0.5 \%$ over these parameters.

and, at the same time, the amplitude of the $2^{\text {nd }}$ mode drops to zero. The fundamental cavity mode thus is created by the constructive interference of basis mode $\psi_{0}$ and $\psi_{2}$ at the cavity center, while the $2^{\text {nd }}$ cavity mode is a result of the destructive interference. The most notable result is that the field amplitude of the $0^{\text {th }}$ cavity mode $\Psi_{0}(0)$ increases by $\approx 50 \%$ at the resonance cavity length in comparison with the equivalent spherical mirror cavity. Since the parameter $g_{0}$ is the same for the spherical and Gaussian mirror cavities for a given cavity length (see equations (1) and (2)), the increase of the field amplitude $\Psi_{0}(0)$ leads to the proportional increase in the coupling strength $g(0)$ of the fundamental cavity mode to a particle trapped at the center. For such a cavity with Gaussian-shaped mirrors, the coupling strength $g(0)$ is thus increased at the resonance by $50 \%$ relative to the equivalent spherical mirror cavity.

\subsection{Coupling between cavity and optical fiber}

One of the advantages of fabricating cavity mirrors on fiber tips is the potential to couple light directly from the fiber into the cavity and vice versa. However, to achieve a high coupling efficiency the spot size as well as the curvature of the wave front of the cavity mode at the mirror should match those of the optical fiber output. To determine the mode overlap we calculate the mode matching integral $Q$ between the fundamental mode $\Psi_{0}(r)$ of the cavity and the output field $F(r)$ of a single-mode fiber,

$$
Q=\frac{\left|\int \Psi_{0}(r) F^{*}(r) r d r\right|^{2}}{\int\left|\Psi_{0}(r)\right|^{2} r d r \int|F(r)|^{2} r d r} .
$$

We assume a Gaussian profile for the transverse mode of the optical fiber with a halfwidth mode waist $\omega_{f}$, which is a good approximation for the fundamental mode of a conventional step index fiber. In our calculations the fiber mode size $\omega_{f}$ varies from $3 \mu \mathrm{m}$ to $12 \mu \mathrm{m}$. The refraction of the optical field on the curved cavity interface is also taken into account in the fiber output field $F(r)$. Figure 8 shows the mode matching integral 


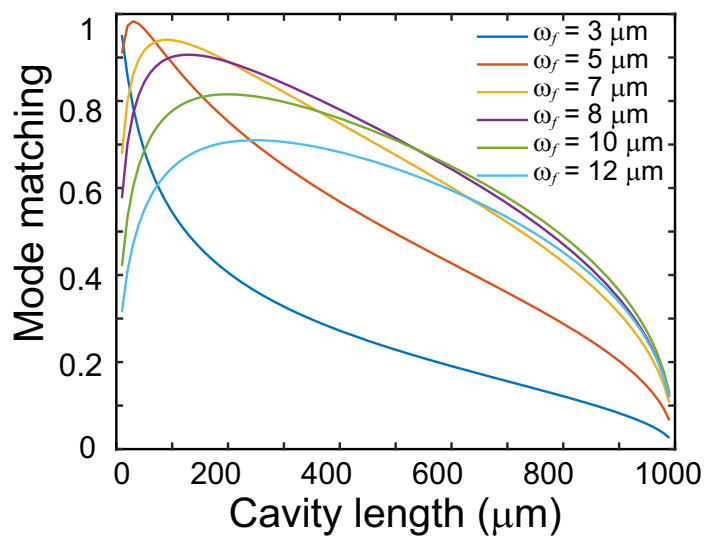

Figure 8. Mode matching integral between the fundamental mode of a spherical mirror cavity with $R=500 \mu \mathrm{m}$ and optical fiber modes with mode half-widths varying from $3 \mu \mathrm{m}$ to $12 \mu \mathrm{m}$.

(a)

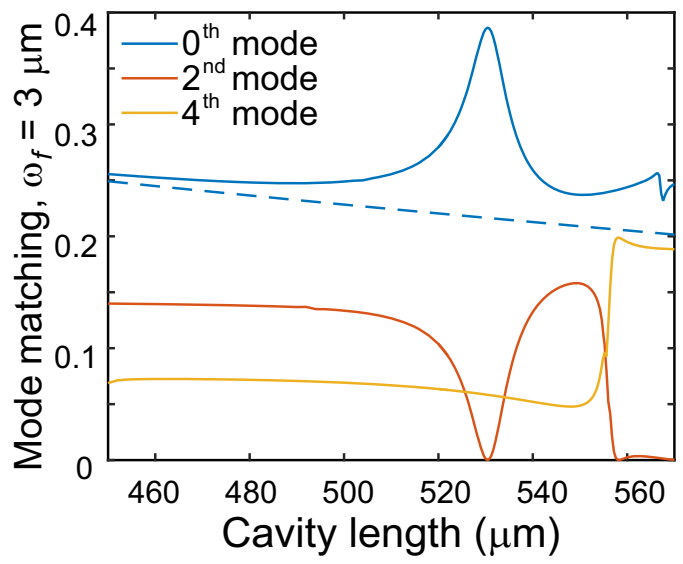

(b)

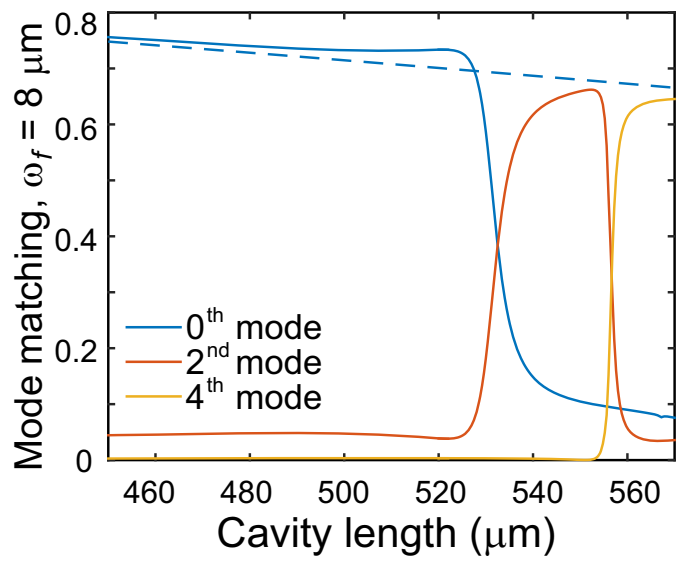

Figure 9. Mode matching integral between the $0^{\text {th }}, 2^{\text {nd }}$ and $4^{\text {th }}$ cavity modes and the fiber modes with the mode half-width of (a) $\omega_{f}=3 \mu \mathrm{m}$ and (b) $\omega_{f}=8 \mu \mathrm{m}$ calculated for a range of the cavity lengths around the resonance at $L=531 \mu \mathrm{m}$. The dashed lines plot the mode matching integral for a cavity with spherical mirrors with $R=500 \mu \mathrm{m}$.

for a cavity with spherical mirrors as a function of the cavity length for a cavity with $R=500 \mu \mathrm{m}$. Mode matching above $95 \%$ is obtained only at small cavity lengths for $w_{f}=5 \mu \mathrm{m}$. As the cavity length increases, the cavity mode field diameter at the mirrors also increases and thus the highest mode-matching coefficient is obtained with a fiber having larger $w_{f}$. However, the fiber mode wave front is concave after being refracted on the curved fiber interface while the cavity mode wave front is convex. For larger mode field diameters this difference in wave front curvature becomes increasingly significant and leads to the overall decrease of the mode matching at longer cavity lengths. For example, at a cavity length of $\sim 500 \mu \mathrm{m}$ a highest mode-matching coefficient around $70 \%$ is observed for $w_{f}=8 \mu \mathrm{m}$.

For cavities with Gaussian mirrors, we concentrate on a cavity with $R=500 \mu \mathrm{m}$, $D=5 \mu \mathrm{m}$ and length around $531 \mu \mathrm{m}$ at which mode mixing occurs. Figure 9 shows the 
mode matching integral calculated for the $0^{\text {th }}, 2^{\text {nd }}$ and $4^{\text {th }}$ eigenmodes of the Gaussian mirror cavity and the fiber modes with $\omega_{f}=3 \mu \mathrm{m}$ and $\omega_{f}=8 \mu \mathrm{m}$ for cavity lengths around the resonance. The figure also shows the mode-matching of a spherical mirror cavity. For a fiber with $\omega_{f}=3 \mu \mathrm{m}$ (which is a typical mode size of a single mode fiber for near infrared wavelengths) the mode matching integral at $L \sim 500 \mu \mathrm{m}$ is below $25 \%$. The cavity mode size on the mirrors at this cavity length is $\sim 12 \mu \mathrm{m}$, much larger than the chosen fiber mode size. At the resonance, however, the mode-matching coefficient increases to almost $40 \%$.

If the fiber has a mode size of $8 \mu \mathrm{m}$, the mode-matching coefficient for the fundamental cavity mode is $\sim 70 \%$ at cavity lengths around $500 \mu \mathrm{m}$, in line with the results for a spherical cavity discussed above. At the resonant cavity length, the coefficient drops below $50 \%$ and continues decreasing as the cavity length increases. At the same time, the mode-matching coefficient of the $2^{\text {nd }}$ order cavity mode increases up to the level of the spherical mirror cavity. Similar behavior is observed between the $2^{\text {nd }}$ and $4^{\text {th }}$ order cavity modes at around $L=555 \mu \mathrm{m}$ where these modes are coupled. However, despite the mode matching decrease around the resonance at $L=531 \mu \mathrm{m}$, just below the resonance in the range of cavity lengths between 528 and $530 \mu \mathrm{m}$ the mode-matching coefficient is still between 70 and $60 \%$ for the $0^{\text {th }}$ order mode. At the same time, the optical field intensity of this mode in the center of the cavity (see figure 7) is 40 to $50 \%$ higher compared to the spherical cavity. For these parameters it is therefore possible to benefit from the enhanced atom-cavity coupling coefficient observed in Gaussian-shaped mirror cavities by having a stronger field in the cavity center while maintaining high cavity-fiber optical coupling.

It is worth noticing that all results presented in this section can easily be generalized to any Gaussian-shaped cavity with different mirror parameters $D$ and $R$. The cavity characteristics can be scaled using the relations defined in Sec. 3.1 and 3.2. If the indentation depth $D$ is different from the one discussed above, the resonant cavity length can be found using figure 5. For a cavity with different radius of curvature $R$, the corresponding cavity length can be obtained from the dependence $L \sim R$, and the optimal fiber mode size from the dependence $\omega_{f} \sim R^{1 / 2}$.

\section{Conclusion}

To summarize, we presented our detailed investigations of the effects of Gaussianshaped mirrors in optical fiber cavities on a range of important parameters. Similarly to spherical mirrors, we found a scaling law for the cavity mode waist and the effective radius of curvature which allows us to determine these parameters from the mirror shape without involved numerical simulations. Using a mode mixing matrix approach, we calculated the effect of the Gaussian mirror shape on the eigenmodes of the cavity and the associated cavity losses. We found that the losses of the fundamental cavity mode increase significantly whenever the cavity mode contains a strong higher order mode component, which happens at discrete "resonant" cavity lengths, a result similar to Ref. 
[17]. However, when the resonance occurs mainly with the $2^{\text {nd }}$ order mode, the losses still remain sufficiently low to allow for high finesse cavities. By choosing an appropriate cavity length, this mode mixing can be utilized to enhance the coupling between the cavity mode and atomic particles trapped at the cavity center. We investigated this effect of the fundamental cavity mode in detail and found a $50 \%$ increase of the cavity coupling through enhancement of the local cavity mode field due to the mode mixing. Furthermore, using the overlap integral between the cavity mode field and the fiber output field, we simulated the mode matching between the two modes with special focus on the lowest cavity eigenmodes around the lowest of these mode mixing resonances. Perhaps surprisingly, the mode matching to a standard single-mode optical fiber improves when the fundamental cavity mode has a significant higher order mode contribution, and it is comparable to the maximum mode matching achievable with spherical mirror fiber cavities using fibers of optimized mode waists.

In conclusion, the coupling between atomic particles and a cavity with Gaussian shaped mirrors shows a significant enhancement due to mode mixing without substantial deterioration of the cavity losses or of the cavity to fiber mode matching. Thus, this effect constitutes a viable method to significantly enhance the interaction between atomic particles and the cavity for relatively long cavity lengths. We therefore envisage that the effect will be particularly useful for the efficient coupling of trapped ions to optical fibers in the context of hybrid quantum information networks $[3,4]$.

\section{Acknowledgements}

We gratefully acknowledge support from EPSRC through the UK Quantum Technology Hub: NQIT - Networked Quantum Information Technologies (EP/M013243/1) and $\mathrm{EP} / \mathrm{J} 003670 / 1$.

\section{References}

[1] Pellizzari T, Gardiner S, Cirac J and Zoller P 1995 Phys. Rev. Lett. 75 3788-3791

[2] Cirac J I, Zoller P, Kimble H J and Mabuchi H 1997 Phys. Rev. Lett. 78 3221-3224

[3] Kimble H J 2008 Nature 453 1023-1030

[4] Monroe C and Kim J 2013 Science 339 1164-1169

[5] Maiwald R, Leibfried D, Britton J, Bergquist C, Leuchs G and Wineland D 2009 Nat. Phys. 5551

[6] Biercuk M J, Uys H, Britton J W, Van Devender A P and Bollinger J J 2010 Nat. Nanotechnol. 5 646-650

[7] Hunger D, Steinmetz T, Colombe Y, Deutsch C, Hänsch T W and Reichel J 2010 New J. Phys. 12065038

[8] Hunger D, Deutsch C, Barbour R J, Warburton R J and Reichel J 2012 AIP Adv. 2012119

[9] Colombe Y, Steinmetz T, Dubois G, Linke F, Hunger D and Reichel J 2007 Nature 450 272-276

[10] Steiner M, Meyer H M, Deutsch C, Reichel J and Köhl M 2013 Phys. Rev. Lett. 110043003

[11] Flowers-Jacobs N E, Hoch S W, Sankey J C, Kashkanova A, Jayich A M, Deutsch C, Reichel J and Harris J G E 2012 Appl. Phys. Lett. 101221109

[12] Brandstätter B, McClung A, Schüppert K, Casabone B, Friebe K, Stute A, Schmidt P O, Deutsch C, Reichel J, Blatt R and Northup T E 2013 Rev. Sci. Instrum. 84123104 
[13] Takahashi H, Morphew J, Oručević F, Noguchi A, Kassa E and Keller M 2014 Opt. Express 22 31317-31328

[14] Ott K, Garcia S, Kohlhaas R, Schüppert K, Rosenbusch P, Long R and Reichel J 2016 Opt. Express 249839

[15] Bick A, Staarmann C, Christoph P, Hellmig O, Heinze J, Sengstock K and Becker C 2016 Rev. Sci. Instrum. 87013102

[16] Gallego J, Ghosh S, Alavi S, Alt W, Martinez-Dorantes M, Meschede D and Ratschbacher L 2016 Appl. Phys. B $\mathbf{1 2 2} 47$

[17] Benedikter J, Hümmer T, Mader M, Schlederer B, Reichel J, Hänsch T W and Hunger D 2015 New J. Phys. 17053051

[18] Harlander M, Brownnutt M, Hänsel W, R and Blatt R 2010 New J. Phys. 12093035

[19] Podoliak N, Takahashi H, Keller M and Horak P 2016 Phys. Rev. Appl. 6044008

[20] Kleckner D, Irvine W T M, Oemrawsingh S S R and Bouwmeester D 2010 Phys. Rev. A 81043814 